\title{
Joint Sparse Spectrum Reconstruction and Information Fusion via $\ell_{1}$-Minimization
}

\author{
Andreas Bollig, Steven Corroy and Rudolf Mathar \\ Institute for Theoretical Information Technology \\ RWTH Aachen University \\ D-52074 Aachen, Germany \\ Email: $\{$ bollig, corroy, mathar $@ @$ ti.rwth-aachen.de
}

\begin{abstract}
This paper considers the problem of sensing a sparsely occupied wideband spectrum utilizing a set of geographically distributed sensing nodes as well as a fusion center. Exchange of measurement data between the sensing nodes and the fusion center takes up parts of the precious radio spectrum and thus, methods for diminishing the minimum amount of measurements still ensuring a reliable reconstruction of the spectrum at the fusion center are needed. To this end we propose two approaches in the form of convex optimization problems to tackle the problem. The first approach applies classic compressive sampling, while the second approach improves the optimization problem, so that all measurements which have been acquired in a distributed manner can be taken into consideration in a single spectrum recovery operation. This makes it possible, to exploit the inherent diversity gain. The presented approaches to efficient distributed spectrum sensing enable reliable dynamic spectrum access.
\end{abstract}

\section{INTRODUCTION}

The radio spectrum is a scarce resource despite its severe underutilization. To solve this problem and make use of the spectral bands which are suitable for radio communication more efficiently, dynamic spectrum access (DSA) has been proposed. DSA is the concept of dynamically allocating parts of a spectral band to users. One of its applications is to allow unlicensed users to access a part of the radio spectrum when it is not being used by its license holder. One of the main requirements of DSA is reliable spectrum sensing, without which the dynamic access of the spectrum inevitably leads to disturbing the licensees usage of its bandwidth. Due to path loss and fading in wireless communication, reliable spectrum sensing can only be accomplished in a distributed manner. However, distributed spectrum sensing makes it necessary to share measurement data, which in turn claims some of the spectral resources that are to be used by actual wireless services. To this end it is desired to minimize the number of measurements which have to be shared for reliable spectrum sensing.

Due to the underutilization of the wireless spectrum, a wideband signal acquired for the purpose of spectrum sensing can be assumed to be only sparsely occupied in the frequency

This work was partly supported by the Deutsche Forschungsgemeinschaft (DFG) project CoSeCo (grant MA 1184/18-1) and by the UMIC research cluster of the RWTH Aachen University. domain, which can be taken advantage of in the process of signal acquisition. The idea of exploiting sparsity in signal recovery has been around for a long time [1]. Due to the advancements in computational power as well as the improvements in optimization algorithms, ambitious computations have become reasonable. Enabled by these developments, a tremendous leap forward has been made in the area of sparse recovery, leading to a new sampling-paradigm called compressive sampling (CS) [2], [3]. Employing the CS theory, the overwhelming amount of sampling-data can be reduced to a number of samples which depends on the amount of information in the signal rather than the maximum possible amount of information the signal can exhibit. In the present field of application, the advent of CS means that a sparse frequency domain signal can be acquired by taking an amount of time-domain samples drastically smaller than necessary in traditional Nyquist-rate sampling and recovering the signal of interest from these.

A rich literature exists in the field of spectrum sensing via compressive sampling. One example of this is the paper by Tian [4] in which the author proposes a system of geographically distributed cognitive radios (CRs) that undersample the spectrum in the time domain and exchange measurement data in a one-hop fashion to benefit from the diversity gain which helps increasing the reliability of the system and fighting channel fading effects. Making use of the distributed average consensus algorithm presented in [5], after a number of iterations each $\mathrm{CR}$ is in possession of the collective view of the spectrum. In [6] the authors of the present work have tackled the problem of spectrum sensing with the newly developed method of matrix completion (MC) [7], [8]. Their system assumes the spectrum occupation to change only slowly and takes advantage of this by introducing a sliding window algorithm that takes measurements of previous sensing cycles into account during the recovery of the current signal.

In this paper, two approaches for recovering the spectrum from an incomplete set of measurements are introduced. The first one is similar to the one introduced in [4] and serves as a benchmark for the second approach. It employs classic compressive sampling and subsequent signal fusion. In contrast to [4], our first approach only considers a single transmitter emitting a signal instead of several ones. In order to reduce 
the necessary amount of known samples even further while still guaranteeing a reliable spectrum recovery, we propose a novel approach. The main contribution of this paper lies in stating an optimization problem for recovering the spectrum in a way that makes it possible to take into account the measurements of all collaborating CRs in a single recovery operation. This unleashes the diversity gain inherent in the samples supplied by the geographically distributed sensing nodes and thus allows us to make optimal use of the available knowledge. Another substantial difference to [4] is, that we evaluate the case where the channel coefficients are known to the system sensing the spectrum. While the authors of [4] also mention this case, they focus on the scenario where the collaborating CRs are not in possession of any channel knowledge.

The remainder of this paper is structured as follows. Section II introduces the mathematical framework which is used throughout the paper. In section III, the two proposed approaches together with their accompanying optimization problems are stated. The two approaches are evaluated by the means of a numerical simulation in section IV and the results are interpreted in section V. Section VI concludes the paper.

\section{AnAlytical Model}

A wide frequency-band containing $n_{\mathrm{sc}}$ non-overlapping subchannels is considered. The sub-channels occupy equal parts of the band. These parts are narrow and thus, the frequencyselective fading can be considered to be flat for each of them.

The licensee of the spectrum, also called primary user, transmits a frequency-domain signal $\mathbf{f} \in \mathbb{C}^{n_{\text {sc }}}$ containing one coefficient per sub-channel. The signal is $k$-sparse, meaning that only $k$ of its $n_{\mathrm{sc}}$ entries are non-zero. The sparsity represents the underutilization of the spectrum. While the partitioning of the band is fixed and known, the amplitudes on the sub-channels change over time.

A group of $n_{\text {cr }}$ geographically distributed cognitive radios is used to sense the signal so that a fusion center can reconstruct the signal $\mathbf{f}$ as observed at the transmitter as accurately as possible from the CR's measurements.

The signal sensed by CR $i$ for $i=1, \ldots, n_{\mathrm{cr}}$ is given by

$$
\tilde{\mathbf{f}}_{i}=\mathbf{H}_{i} \mathbf{f}+\mathbf{n}_{i}
$$

where $\mathbf{H}_{i} \in \mathbb{C}^{n_{\mathrm{sc}} \times n_{\mathrm{sc}}}$ is a diagonal channel matrix, the entries of which are distributed according to a Rayleigh distribution. The entry $\left[\mathbf{H}_{i}\right]_{j j}$ represents the signal attenuation that $\mathrm{CR} i$ experiences on channel $j$. The entries of the noise vector $\mathbf{n}_{i}$ are Gaussian independently and identically distributed with mean zero and variance $\sigma^{2}$. In this work, the CRs are assumed to have knowledge of their respective channel matrix $\mathbf{H}_{i}$.

In order to keep the amount of sampled data which has to be sent to the fusion center as low as possible, the CRs employ compressive sampling, meaning that they undersample the spectrum in the time domain, i.e., CR $i$ takes $m \ll 2 n_{\text {sc }}$ randomly distributed samples of the time-signal vector

$$
\tilde{\mathbf{t}}_{i}=\mathbf{F}_{2 n_{\mathrm{sc}}}^{-1} \mathbf{D}_{n_{\mathrm{sc}}} \tilde{\mathbf{f}}_{i}
$$

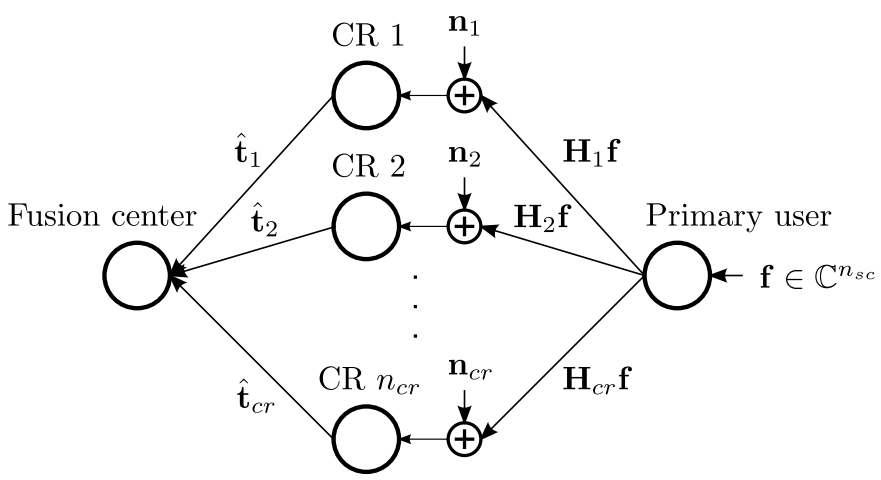

Fig. 1. System model.

For simplicity, we consider the observed channels to be located at the baseband. Hence, the vector containing the discrete Fourier transform (DFT) coefficients is symmetric. The matrix $\mathbf{D}_{n_{\mathrm{sc}}} \in\{0,1\}^{2 n_{\mathrm{sc}} \times n_{\mathrm{sc}}}$ assembles the symmetric frequencydomain vector from the channel coefficients found in $\tilde{\mathbf{f}}_{i}$. An example of $\mathbf{D}$ for $n_{\mathrm{sc}}=3$ is given by

$$
\mathbf{D}_{3}=\left(\begin{array}{lll}
0 & 0 & 0 \\
1 & 0 & 0 \\
0 & 1 & 0 \\
0 & 0 & 1 \\
0 & 1 & 0 \\
1 & 0 & 0
\end{array}\right) .
$$

$\mathbf{F}_{2 n_{\mathrm{sc}}}^{-1}$ stands for the inverse discrete Fourier transform (IDFT) matrix of size $2 n_{\mathrm{sc}}$. Each CR transmits $m$ time-domain samples to the fusion center. These are selected in the following way:

$$
\hat{\mathbf{t}}_{i}=\mathbf{M}_{i} \tilde{\mathbf{t}}_{i}
$$

where $\mathbf{M}_{i} \in\{0,1\}^{m \times 2 n_{\mathrm{sc}}}$ denotes the selection matrix consisting of $m$ randomly chosen rows of the $2 n_{\mathrm{sc}} \times 2 n_{\mathrm{sc}}$ identity matrix. Note, that all matrices $\mathbf{M}_{i}$ are known to the fusion center.

In order to clarify the notations used in the present paper, we illustrate the considered setup in Fig. 1.

\section{OPTIMIZATION PROBLEMS}

To reach the collective goal, i.e., reconstructing $\mathbf{f}$ from the measurements, we propose two approaches, both of which employ $\ell_{1}$-minimization.

\section{A. First Approach: Separate Reconstruction and Fusion}

In the first approach, basic compressive sampling is applied to the sampled data of each CR separately. This approach is similar to the one proposed in [4]. In this work, it serves as a benchmark against which our novel approach is compared. For the data received from $\mathrm{CR} i$, the fusion center solves the following convex optimization problem:

$$
\begin{aligned}
& \underset{\mathbf{f}_{i}}{\operatorname{minimize}}\left\|{\stackrel{\circ}{\mathbf{f}_{i}}}_{i}\right\|_{\ell_{1}} \\
& \text { subject to }\left\|\mathbf{M}_{i} \mathbf{F}_{2 n_{\mathrm{sc}}}^{-1} \mathbf{D}_{n_{\mathrm{sc}}} \mathbf{H}_{i} \stackrel{\circ}{\mathbf{f}}_{i}-\hat{\mathbf{t}}_{i}\right\|_{\ell_{2}} \leq \epsilon_{\mathrm{s}}
\end{aligned}
$$


where $\|\cdot\| \ell_{\ell_{1}}$ is the $\ell_{1}$-norm defined by $\|\mathbf{z}\|_{\ell_{1}}=\sum_{j=1}^{n_{\mathrm{z}}}\left|z_{j}\right|$ given some vector $\mathbf{z} \in \mathbb{C}^{n_{\mathrm{z}}}$ and $\|\cdot\|_{\ell_{2}}$ stands for the $\ell_{2}$-norm, i.e., $\left.|| \mathbf{z}\right|_{\ell_{2}}=\sum_{j=1}^{n_{\mathrm{z}}}\left|z_{j}\right|^{2}$. The constant $\epsilon_{\mathrm{s}}$ bounds the amount of expected noise energy in the signal.

The reconstructed spectra $\mathbf{f}_{i}^{\star}$ are subsequently fused by the means of equal gain combining:

$$
\stackrel{\circ}{\mathbf{f}}=\frac{1}{n_{\mathrm{cr}}} \sum_{i=1}^{n_{\mathrm{cr}}} \stackrel{\circ}{\mathbf{f}}_{i}^{\star} .
$$

Here, the star $\star$ denotes the solution to an optimization problem, i.e., $\stackrel{f}{i}_{i}^{\star}$ is the solution to (5). The vector $\mathbf{f}^{\circ}$ is the reconstruction of the frequency-domain signal as observed at the transmitter, i.e., in a scenario with $\mathbf{n}_{i}=\overrightarrow{0}$ for all $i$, a perfect reconstruction would yield $\stackrel{\circ}{\mathbf{f}}=\mathbf{f}$.

\section{B. Second Approach: Combined Reconstruction}

In the new approach, all available measurements are considered in a single combined reconstruction of the spectrum in order to make better use of the diversity gain inherent in the available data. Instead of solving the optimization problem separately for each CR's data, the optimization problem has been modified so that the $\ell_{1}$-minimization is used to directly recover the primary user's signal from all spectral measurements the fusion center has received.

In order to do so, we first define the matrix of time domain samples given by

$$
\tilde{\mathbf{T}}=\left[\tilde{\mathbf{t}}_{1}, \ldots, \tilde{\mathbf{t}}_{n_{\mathrm{cr}}}\right]
$$

in which each column represents the time-domain signal as observed at one of the CRs. Furthermore we define the sampling set $\Omega$. It has the cardinality $m$ and is comprised of the indices of the entries of $\tilde{\mathbf{T}}$ that are to be sampled by the respective CRs. We define the sampling operator $\mathcal{P}_{\Omega}: \mathbb{R}^{2 n_{\text {sc }} \times n_{\text {cr }}} \rightarrow \mathbb{R}^{2 n_{\text {sc }} \times n_{\text {cr }}}$ as follows. Given a matrix $\mathbf{X}$,

$$
\left[\mathcal{P}_{\Omega}(\mathbf{X})\right]_{i j}= \begin{cases}{[\mathbf{X}]_{i j}} & \text { if }(i, j) \in \Omega \\ 0 & \text { otherwise }\end{cases}
$$

Making use of the previous definitions, the measurements available at the fusion center can be written as

$$
\hat{\mathbf{T}}=\mathcal{P}_{\Omega}(\tilde{\mathbf{T}}) .
$$

Finally we define the concatenated channel matrix as

$$
\overline{\mathbf{H}}=\left[\mathbf{H}_{1}, \ldots, \mathbf{H}_{n_{\mathrm{cr}}}\right] .
$$

The resulting convex optimization problem which has to be solved by the fusion center is given by

$$
\begin{aligned}
& \underset{\mathfrak{f}}{\operatorname{minimize}}\|\stackrel{\mathbf{f}}{\mid}\|_{\ell_{1}} \\
& \text { subject to }\left\|\mathcal{P}_{\Omega}\left(\mathbf{F}_{2 n_{\mathrm{sc}}}^{-1} \mathbf{D}_{n_{\mathrm{sc}}} \overline{\mathbf{H}}\left(\mathbf{I}_{n_{\mathrm{cr}}} \otimes \stackrel{\circ}{\mathbf{f}}\right)\right)-\hat{\mathbf{T}}\right\|_{\ell_{2}} \leq \epsilon_{\mathrm{c}} \text {, }
\end{aligned}
$$

where $\mathbf{I}_{n_{\mathrm{cr}}}$ is the identity matrix of size $n_{\mathrm{cr}}$ and $\otimes$ denotes the Kronecker product. Note that since this optimization problem takes into account the measurements of all CRs, no fusion is necessary and $\mathbf{f}$ is the final reconstruction of the primary user's signal.
Since both optimization problems are convex, they can be efficiently solved computationally. Note, that for a single cognitive radio, the optimization problems of the two approaches are equivalent.

\section{NUMERICAL EVALUATION}

The two approaches have been compared via simulation, employing the parameters given in table I. For the purpose of solving the convex optimization problems, the CVX software package [9] has been used.

For simplicity, the real - and imaginary parts of the nonzero entries of $\mathbf{f}$ are generated randomly from a uniform distribution. The channel coefficients are distributed according to a Rayleigh distribution with standard deviation $\gamma=0.5$ and the noise vectors contain entries which are distributed according to a zero-mean Gaussian distribution. The variance of the noise values is defined by the respective SNR, where the SNR is defined as the signal energy of the wideband signal over the entire spectrum divided by the overall noise energy (again over the entire spectrum).

Throughout this work, the samples are counted in number of samples per reconstruction, i.e., we have a fixed number of samples per optimization problem being solved, such that the system applying the first approach takes an overall number of $m \cdot n_{\text {cr }}$ samples since a signal recovery is done for each CR, while the system applying the second approach only takes $m$ samples overall, i.e., $\mathrm{m} / n_{\text {cr }}$ samples per CR. For analyzing the results, it is important to understand that in all simulations the second approach takes $n_{\text {cr }}$ times less samples than the first approach.

TABLE I

SCENARIO PARAMETERS

\begin{tabular}{lcc}
\hline Parameters & Symbol & Value(s) \\
\hline Number of frequency subchannels & $n_{\mathrm{sc}}$ & 100 \\
Number of busy subchannels & $k$ & 5 \\
Number of CRs & $n_{\mathrm{cr}}$ & $\{1,3,5,7,9\}$ \\
Signal to noise ratio (SNR) & $\mathrm{SNR}$ & $\{0,5,10, \ldots, 40\} \mathrm{dB}$ \\
Number of samples per reconstruction & $m$ & $\{10,20,30, \ldots, 100\}$ \\
\hline
\end{tabular}

In our scenario, we define the root mean square error (RMSE) by

$$
e_{\mathrm{RMSE}}=\sqrt{\frac{\|\mathbf{f}-\stackrel{\circ}{\mathbf{f}}\|_{\ell_{2}}}{n_{\mathrm{sc}}}} .
$$

The RMSE caused by undersampling the signal with 30 and 100 of the 200 Nyquist-rate samples respectively is depicted in Fig. 2 and Fig. 3.

The two figures show the resulting RMSE for various numbers of collaborating CRs with respect to the SNR. Note that the two approaches are equivalent for the case where only a single cognitive radio is utilized and that therefore the corresponding curves in the graph completely overlap, rendering the one of the separate approach invisible.

As expected, decreasing the amount of noise in the system yields a better reconstruction result in all situations since the reconstruction is compared to the original noiseless primary 


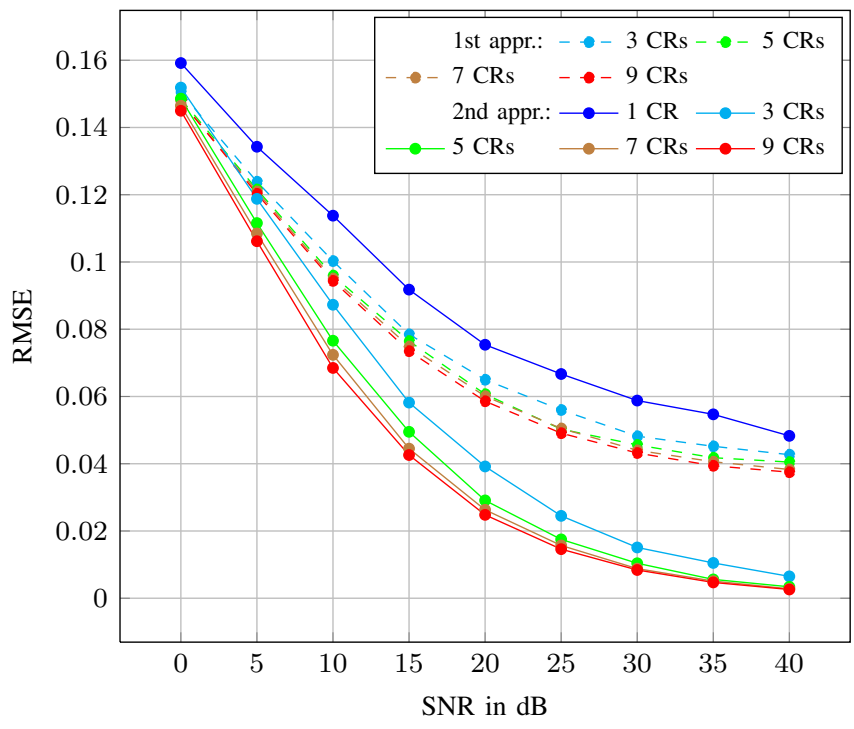

Fig. 2. RMSE of reconstructing the primary user's signal utilizing various numbers of collaborating CRs taking 30 samples per reconstruction.

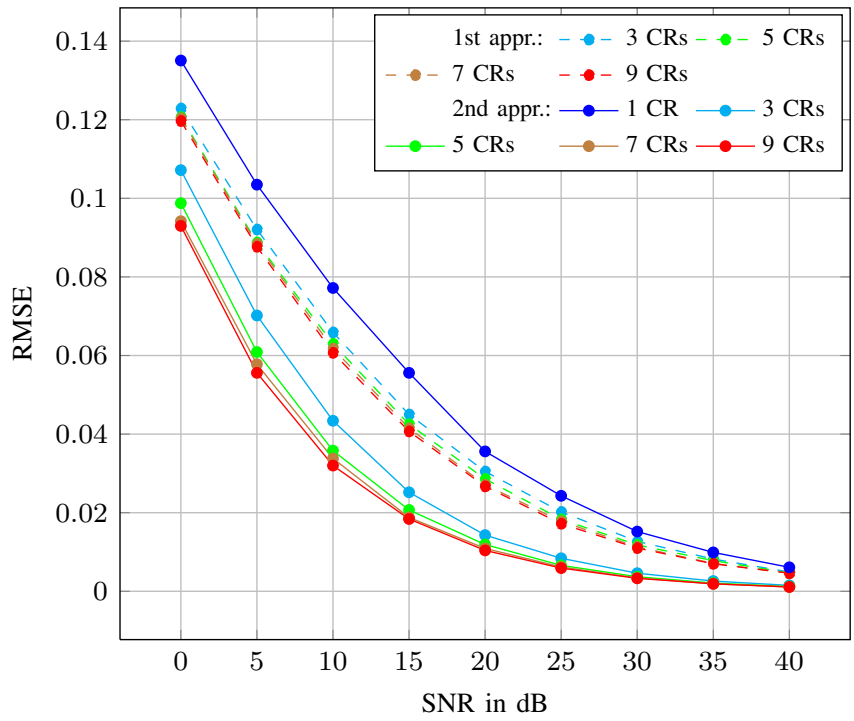

Fig. 3. RMSE of reconstructing the primary user's signal utilizing various numbers of collaborating CRs taking 100 samples per reconstruction.

user signal. However, in the heavily undersampled case, i.e., 30 samples per signal recovery, the RMSE resulting from applying the separate reconstruction and subsequent fusion decreases much slower for decreasing noise than the RMSE of the second approach. Considering the much lower number of overall samples taken for method two, e.g., in Fig. 2 the second approach utilizes an overall number of 30 samples in all curves, while the first approach takes $\{30,90,150, \ldots\}$ samples for $\{1,3,5 \ldots\} \mathrm{CRs}$, it is astonishing to see, that the combined reconstruction still performs better for all considered parameter sets.

In Fig. 4 we observe how the error-behavior of recovering the primary user's signal depends on the number of samples that are taken into consideration per reconstruction. For this

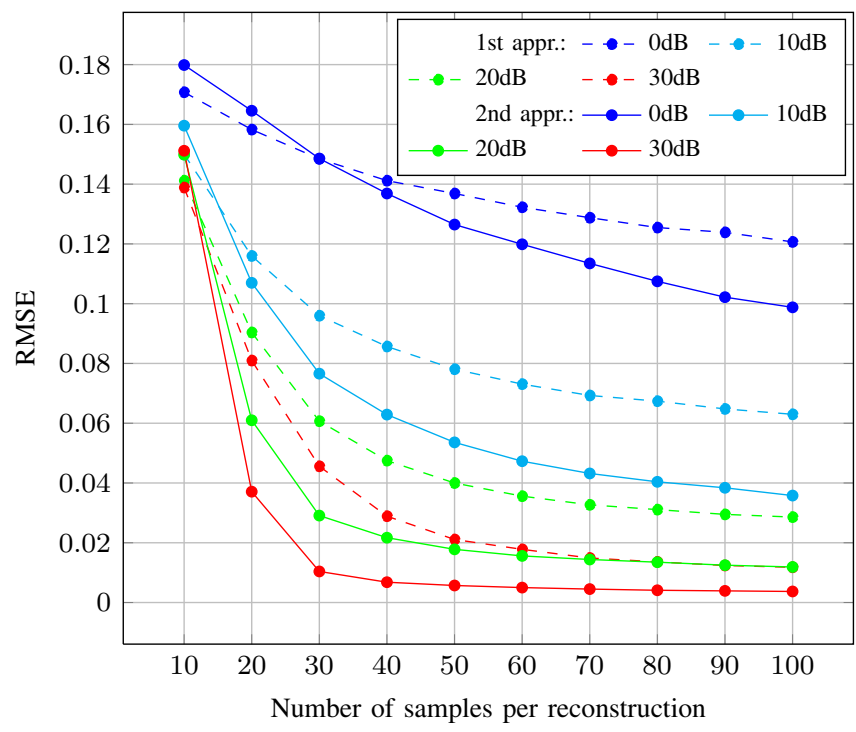

Fig. 4. RMSE of reconstructing the primary user's signal for various SNRs utilizing 5 collaborating CRs.

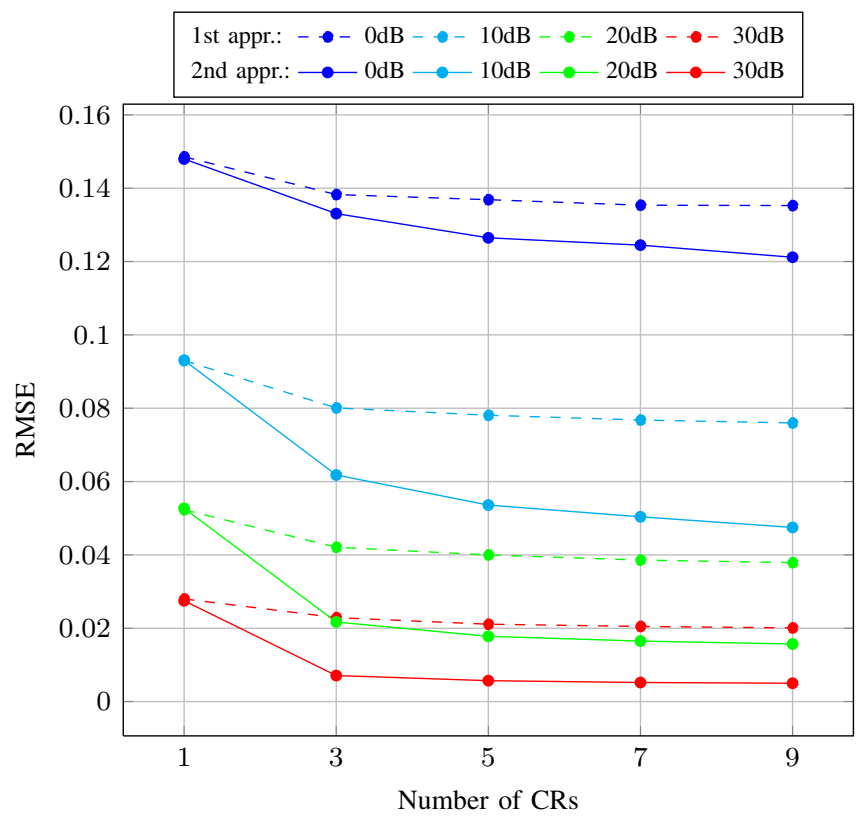

Fig. 5. RMSE of reconstructing the primary user's signal for various SNRs taking 50 samples per reconstruction.

we make use of a fixed number of cognitive radios and varying signal to noise ratios.

For each SNR, two performance regions can be defined. The threshold value dividing the regions gets smaller for an increasing SNR. For a number of samples smaller than the threshold, the first approach performs better, while the second approach performs better for a number of samples higher than the threshold.

Fig. 5 shows how the two introduced approaches perform when the number of collaborating CRs changes while the number of samples per reconstruction is fixed at 50. Again, 
since the two methods are equal for a single cognitive radio, their error performance is the same in this case. However, when the number of collaborating CRs is increased, the method employing the combined reconstruction constantly outperforms the method doing the reconstruction separately despite the large difference in the number of overall considered samples.

\section{AnAlysis of the Results}

Considering the big difference in the overall number of samples taken in the different methods, the numerical evaluation shows vast RMSE improvements when employing the second - rather than the first approach to signal reconstruction. In this section we make an attempt at identifying and explaining the effects leading to the results on hand.

The first effect is found in the observations depicted in Fig. 6. The figure shows the error performance of the reconstruction from measurement data of a single CR in different SNR regions. As one would obviously expect, increasing the number of samples available for the signal recovery decreases the RMSE. However, an interesting observation directly visible in the figure is, that in terms of decreasing the RMSE, the more samples we take into consideration the less gain we experience from every additional sample. Although the number of samples used for the signal recovery increases by 10 from line to line, the distance between two adjacent lines becomes smaller as the number of samples goes up.

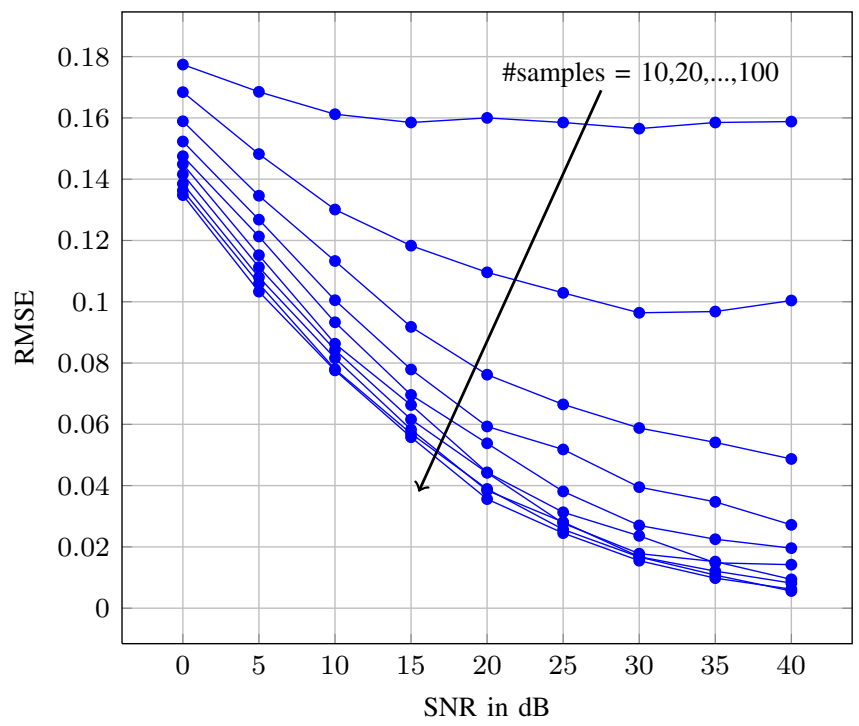

Fig. 6. RMSE of reconstructing the primary user's signal from the measurements of a single CR utilizing various numbers of samples.

In reverse this means, that the first samples are the most valuable ones.

In the first approach, a lot more samples are available, however as we have seen above, the higher the number of samples per reconstruction, the lower the error gain per sample and thus, the disadvantage of the second approach caused by a much lower amount of available time measurements is smaller than may be expected.

Furthermore, the reconstruction of the spectral coefficients using the combined knowledge of all CRs yields a high diversity gain in contrast to the separate reconstruction and subsequent fusion. The reason for this is, that in the separate reconstruction, the diversity gain only kicks in at the fusion stage while in the combined reconstruction the whole recovery process gains from all available information.

Considering the combination of these two effects, namely the diminishing returns in terms of error gain despite of an increased number of available samples and the lower diversity gain of the first approach, the second approach can be expected to have a greatly enhanced performance which is in line with the numerical results.

\section{CONCLUSION}

In this work we have proposed two approaches for the purpose of reducing signaling overhead between a set of geographically distributed cognitive radios and a fusion center which collaboratively try to estimate a signal transmitted by a primary user. Both recovery algorithms have been formulated as convex optimization problems in order to make the signal reconstruction from a small subset of time-domain samples tractable, where the first approach applies actual compressive sampling and the second approach consists of a modified optimization problem designed to reduce the number of necessary samples even further. Both approaches have been shown to be viable through a numerical simulation, the results of which have been discussed for their different dimensions. Finally an attempt has been made to explain the outcomes. As a future research direction, we plan to implement both presented approaches on a software defined radio platform to evaluate their performance in a realistic setting.

\section{REFERENCES}

[1] D. L. Donoho, "Scanning the technology," Proceedings of the IEEE, vol. 98, no. 6, pp. 910-912, Jun. 2010.

[2] —_ "Compressed sensing," Information Theory, IEEE Transactions on, vol. 52, no. 4, pp. 1289-1306, Apr. 2006.

[3] E. J. Candès, J. Romberg, and T. Tao, "Robust uncertainty principles: Exact signal reconstruction from highly incomplete frequency information," Information Theory, IEEE Transactions on, vol. 52, no. 2, pp. 489-509, Feb. 2006.

[4] Z. Tian, "Compressed wideband sensing in cooperative cognitive radio networks," in Global Telecommunications Conference, 2008. IEEE GLOBECOM 2008. IEEE, Nov. 2008, pp. 1-5.

[5] L. Xiao, S. Boyd, and S.-J. Kim, "Distributed average consensus with least-mean-square deviation," Journal of Parallel and Distributed Computing, vol. 67, no. 1, pp. 33 - 46, Jan. 2007.

[6] S. Corroy, A. Bollig, and R. Mathar, "Distributed sensing of a slowly time-varying sparse spectrum using matrix completion," in IEEE International Symposium on Wireless Communication 2011, Nov. 2011, pp. 296-300.

[7] E. J. Candès and Y. Plan, "Matrix completion with noise," Proceedings of the IEEE, vol. 98, no. 6, pp. 925-936, Jun. 2010.

[8] R. Keshavan, A. Montanari, and S. Oh, "Matrix completion from noisy entries," The Journal of Machine Learning Research, vol. 99, pp. 20572078, Aug. 2010.

[9] M. Grant and S. Boyd, "CVX: Matlab software for disciplined convex programming, version 1.21," http://cvxr.com/cvx, Apr. 2011. 